\title{
Récits sériels exploratoires
}

Exploratory Serial Narratives

\section{Marta Boni et Camille Martinez}

\section{(2) OpenEdition \\ Journals}

Édition électronique

URL : http://journals.openedition.org/pratiques/5876

DOI : 10.4000/pratiques.5876

ISSN : 2425-2042

\section{Éditeur}

Centre de recherche sur les médiations (CREM)

\section{Référence électronique}

Marta Boni et Camille Martinez, «Récits sériels exploratoires », Pratiques [En ligne], 181-182 | 2019,

mis en ligne le 30 juin 2019, consulté le 20 juillet 2019. URL : http://journals.openedition.org/ pratiques/5876 ; DOI : 10.4000/pratiques.5876

Ce document a été généré automatiquement le 20 juillet 2019.

(c) Tous droits réservés 


\title{
Récits sériels exploratoires
}

\author{
Exploratory Serial Narratives
}

\author{
Marta Boni et Camille Martinez
}

1 Étudier le récit à l'ère contemporaine, numérique et marquée par une multiplicité de supports médiatiques, signifie se mesurer à la narration sérielle, de plus en plus présente sur tout type d'écran et notamment à la télévision (la télévision traditionnelle linéaire ou les nouveaux fournisseurs de services comme Netflix et Amazon). Cette sérialité, que les spectateurs dévorent à longueur de journée, de mois, d'année, a encouragé plusieurs chercheure's à penser le récit d'abord en termes de temps. Les analystes des séries télévisées s'accordent en effet sur le fait que celles-ci sont des objets audiovisuels très ou trop longs, voire "potentiellement interminables » (Esquenazi, 2014, p. 140), denses en détails et morcelés. À une observation des phénomènes de construction de mondes contemporains, cependant, il apparait qu'une perspective en termes d'espace serait plus pertinente, afin d'étudier les spécificités des séries télévisées qui forment des récits évolutifs, multiformes, ouverts et articulés en plusieurs fragments.

2 L'étude de la temporalité des récits sériels permet de mettre en évidence l'évolution des personnages et leur psychologie (Martin, 2014 ; Jost, 2015), la répétition et la variation d'éléments textuels (Esquenazi, 2014; Soulez, 2011), la complexité de tels moments narratifs et leur « pyrotechnie » (Mittell, 2006, p. 35), leur architecture et leur propension à imiter le vivant (Pescatore \& Innocenti, 2012), leur relation avec la clôture textuelle (Dubois, 2015 ; Lifschutz, 2018) et la façon qu'ont les séries de constamment repousser la clôture narrative, afin de prolonger dans le temps leur existence. La dernière mouture du Récit cinématographique d'A. Gaudreault et F. Jost (2017) inclut des analyses de séries en plus des analyses de films et se concentre sur l'identification des actions et des temporalités d'une série, sur le décryptage des façons par lesquelles la série est racontée, par rapport à la construction des évènements diégétiques.

3 Il est donc clair que la série existe dans le temps et que sa durée est aussi un signe de son succès commercial. Mais si nous voulons considérer la relation qu'entretiennent les spectateurs avec le texte sériel, notamment à l'époque du numérique, marquée par un éloignement des rythmes imposés de la grille des programmes télévisuelle ainsi que par 
une prolifération de supports et de récits, le temps n'est pas la seule dimension (et approche) possible. Dans la perspective que nous proposons, qui allie analyse esthétique, narratologie et pragmatique de la réception, nous comparons les séries télévisées à des mondes qui, savamment esquissés dans le pilote, s'étoffent de détails au fil des épisodes, rendant accessible aux spectateurs un territoire qui, lorsque la série rencontre le succès, se montre soit très complexe, soit possède des personnages attachants et rassurants, ou permet de retrouver ces deux caractères réunis. Autrement dit, nous proposons d'analyser la série comme un espace, pluridirectionnel, élargi, explorable, comme un monde ou un état des choses, ces choses étant des objets, des situations, des personnages, des lois naturelles, etc.

4 Bien que des approches thématiques du concept d'espace (espaces surveillés, traversés, sécurisés et contrôlés, road movies, conquête d'un territoire, espace de la ville, espace et genre) soient possibles, la présente contribution propose d'analyser l'espace fictionnel des séries en tant qu'espace qui se donne comme point de départ pour des explorations multiples. Notre position consiste à soustraire l'espace fictionnel à sa simple fonction de décor, en le considérant comme spécifique au médium, esthétiquement et narrativement déterminant dans le développement d'une série, tout en complexifiant l'idée selon laquelle ce serait uniquement la longueur textuelle, ou le temps, qui permettrait l'expansion d'une série.

\section{La triple temporalité - explorer le temps?}

5 C'est l'approche temporelle qu'a privilégiée J.-P. Esquenazi en 2014 dans L'Art des séries télé . Il y écrit que " la série est un art du temps » en expliquant que les histoires s'y racontent sur une longue durée et font par conséquent appel à la mémoire personnelle et collective (ibid., p. 140)ํㅗㄴ Les séries, telles des horloges suisses, sont des "machines à scénarios » inépuisables (ibid., p. 161). Elles sont réglées par une formule à la triple fonction: délimiter la narration, le style et l'univers fictionnel. Trois ans plus tard, J.-P. Esquenazi convient que l'idée de formule s'applique très bien pour les séries épisodiques - c'est-àdire exposant une nouvelle narration à chaque épisode - mais se heurte à sa propre limite lorsqu'il s'agit de narrations labyrinthiques et entremêlées. Il enrichit alors sa position en y articulant une approche partiellement spatiale, s'appuyant sur les outils d'analyse de la création de mondes fictionnels élargis et notamment les théories de L. Doležel. Pour lui « il est bien évident que le travail du texte est essentiel au processus d'accroissement de l'univers fictionnel. Apportant sans cesse de nouveaux événements, de nouveaux personnages, explorant de nouvelles zones du temps et de l'espace de l'univers fictionnel, il complexifie, complète, augmente, enrichit celui-ci » (Esquenazi, 2017, p. 91). L'outil mobilisé, bien que considérant temps et espace dans une inséparabilité constitutive, reste toutefois au service d'une vision principalement temporelle de la série télé, cherchant à expliquer comment celle-ci articule un montage audiovisuel (deleuzien et profondément lié au temps) et un «montage narratif » dont l'analyse permet de capture l'« image du temps » d'une série (ibid., p. 161-184).

Dans ce cadre, remarquons que la temporalité sérielle est au moins triple car elle fait se superposer trois segments temporels distincts et connectés: le temps diégétique, le temps narratif et le temps spectatoriel. Le premier, le temps diégétique, est vécu par les personnages. C'est la période de temps sur laquelle se déroulent les récits, ceux-ci pouvant s'écouler sur une journée ou plusieurs années. Dans certaines séries, ce temps 
constitue le cadre narratif ou le concept soutenant l'œuvre. C'est le cas dans La Casa de Papel (Antena 3, 2017, Netflix, 2019-) dont les récits entremêlés se déroulent sur douze jours pendant lesquels braqueurs et otages sont confinés dans la Fabrique nationale de la monnaie de Madrid. L'espace y étant clos et quasi-unique, c'est à première vue le temps qui marque l'évolution narrative en s'affichant directement à l'écran. Il balise une narration qui lui est dévolue et dépendante et dont découlent les choix esthétiques de postproduction comme les surimpressions de données temporelles extradiégétiques. Dans Narcos (Netflix, 2015-2017) à l'inverse, les deux premières saisons racontent l'intégralité de la vie du cartel de Medellín, jusqu'à la mort de Pablo Escobar et se déroulent sur plusieurs décennies. Le temps diégétique y est élastique et généralement esthétisé.

7 Cette première temporalité s'articule et s'adapte au temps narratif, le nombre d'heures que prend la narration pour se dérouler. Dans le cas de La Casa de Papel, il représente environ 13 heures de visionnement découpées en 15 épisodes. Certaines séries comme $24 \mathrm{~h}$ Chrono (Fox, 2001-2010, puis 2014) font parfaitement coïncider le temps diégétique et le temps narratif en utilisant cette caractéristique temporelle non plus comme une contrainte mais comme un levier narratif, en l'intégrant à la narration. 24h Chrono raconte vingt-quatre heures de la vie de ses personnages en vingt-quatre heures de narration, découpées en vingt-quatre épisodes.

8 Ces deux temps superposés s'écoulent quant à eux dans un temps que nous appelons spectatoriel, car il correspond au temps réel vécu par le spectateur de sa découverte du premier épisode à la révélation du dernier. Cette temporalité est tributaire du rythme de diffusion du programme, déterminé et organisé par les grilles horaires des chaines de télévision et/ou la rapidité de sa production et ses hiatus. Sa cadence peut être quotidienne ou hebdomadaire et peut suivre des cycles annuels ou pluriannuels. Ainsi, 24h Chrono dans sa formule originale et imaginée par Fox - c'est-à-dire non visionné en rafale après diffusion - fait coïncider l'heure vécue par les personnages avec l'heure vécue par le spectateur (où 1 heure de visionnage est à concevoir en tant que narration purement télévisuelle, incluant donc les pauses publicitaires - comme le remarque J. Ellis [2011], la même série vue en DVD, donc sans annonces, devrait s'appeler 18h Chrono). Elle ne va pas cependant jusqu'à faire coïncider parfaitement les temps narratif et spectatoriel puisqu'elle ne diffuse pas ses saisons sur vingt-quatre heures. Le temps spectatoriel de diffusion représente au contraire vingt-quatre semaines de la vie de ses spectateurs. La série anglaise Sherlock (BBC One, 2010-), quant à elle, a laissé s'écouler jusqu'à trois ans entre la diffusion de sa saison 2 et celle de sa saison 3.

Il est vrai que ces trois temporalités coexistent également au cinéma et en littérature : toutefois, la singularité du médium télévisuel tient en ce que son lien aux temporalités décrites apparait marqué, intime et esthétiquement déterminant puisqu'il implique une plus grande élasticité du temps, tout en générant un rendez-vous ritualisé et un investissement accru de la part du spectateur. Aussi, si elle permet de comprendre la sérialité télévisuelle classique, la perspective purement temporelle révèle quelques limites par rapport à la consommation télévisuelle de l'époque des plateformes over the top et du visionnage en rafale. Les pratiques et rythmes personnels de consommation des spectateurs complexifient les spécificités observables d'une série télévisée, comme le soulignent d'ailleurs les remarques sur la télévision complexe (Mittell, 2015). La complexité et l'élasticité du temps nous révèlent aussi que, pour faire sens d'une multiplicité de couches superposées, il est possible de penser les séries comme des espaces. 


\section{L'approche spatiale}

l'ère de Netflix, Hulu et Amazon, et donc des limites des remarques sur la coïncidence des trois temporalités, certaines études récentes ont proposé une alternative spatiale à l'analyse du temps des séries. À travers le déploiement de ses artifices sur plusieurs épisodes navigables, toujours présents sous la main des spectateurs connectés, le récit produit un espace. En même temps, l'activité des spectateurs, tout en se déroulant sur un temps (qui appartient cette fois aux spectateurs eux-mêmes et pas à la grille des programmes) correspond à l'exploration d'un espace. Si nos analyses précédentes se concentrent sur un point de vue principalement transmédiatique (Boni \& Berton, 2019), dans le présent article on propose une lecture spatiale de la série avec une attention pour la dimension esthétique et narrative, mettant en évidence les récits que nous définissons comme exploratoires. Notre hypothèse est que la notion d'espace permet de comprendre les mécanismes d'extension/expansion opérés par les séries télé. Il s'agira de définir une forme d'immersion en passant de l'angle temporel - le fait de renouer régulièrement avec un contrat fictionnel passé précédemment - à l'angle spatial - le fait de replonger dans un monde fictionnel habitable dans lequel on peut aller et venir au gré de l'immersion.

11 Certains nouveaux objets sériels incarnent des logiques qui sont clairement davantage spatiales que temporelles. Comment analyser des séries aux saisons courtes aux récits clos, aux histoires bouclées telles que les anthologies? Dans ce cas, la perspective spatiale semble plus prometteuse. À titre d'exemple, la série Fargo (FX, 2014-) renouvelle chaque saison son casting tout en connectant ses fragments par un sens plus large en gardant son unité de lieu, le Midwest américain. True Detective (HBO, 2014-), quant à elle, déplace l'espace narratif de la Louisiane (dans la première saison) au nord de la Californie (dans la seconde) conservant son thème, son ton et son traitement comme pierres angulaires. La série anthologique Black Mirror (Channel 4 2011-2014; Netflix 2016-) adopte ce même modèle et transporte ses récits d'un lieu à l'autre mais l'applique au rythme épisodique comme le faisait Alfred Hitchcock Presents (CBS 1955-1960 ; NBC 1960-1962) dans les années 1950 et non au rythme saisonnier. Ces séries peuvent pourtant être elles aussi potentiellement inépuisables et vivre «selon un mode de narration infini » (Esquenazi, 2014, p. 140). L'ancrage de chaque saison dans des espaces fictionnels différents devient leur point commun: l'espace peut y être analysé comme moteur narratif et vecteur d'infinité potentielle de la collection de récits. Une approche spatiale, étroitement liée à une approche temporelle, devient alors utile.

12 Suivre une série télé n'impliquerait pas simplement de découvrir des récits se déroulant au premier plan d'un décor fictif, mais se familiariser avec un espace fictionnel à la croisée du concret et de l'abstrait duquel naissent, dépendent et se pérennisent les récits sériels. L'approche d'A. Gardies est utile en ce sens. Pour l'auteur, au cinéma les récits sont tributaires de l'espace dans lequel ils se déroulent. C'est le cas du Salaire de la peur (Clouzot, 1953) dans lequel l'environnement géographique (le nord de l'Afrique), culturel (musiques, langues), politique (occupation du territoire par l'armée française) et architectural (ville coloniale) constitue un moteur narratif important, tantôt opposant, tantôt allié, toujours adjuvant (Gardies, 1993, p. 107-136).

13 Selon la théorie cinématographique spatiale défendue par A. Gardies (ibid., p. 102), c'est l'ensemble du « système relationnel » entre l'espace et les personnages qui est vecteur de 
récit. Ce système, l'espace-lieu comme principal « actant » est appelé « espace narratif ». Dans cette perspective « ce qui fonde le réseau des déplacements du héros, c'est moins le déterminisme de la consécution que la nécessité de se rendre en des lieux spécifiques pour y acquérir les valeurs dont il a besoin pour sa quête » (ibid., p. 149). A. Gardies affirme que tout récit raconte, au fond, l'histoire d'un sujet dans ses rapports à un espace. Cet angle spatial, articulé à la spécificité d'une série qui multiplie les lieux et les lie ou non les uns aux autres, permet de préciser les détails de l'expansion temporelle (récits et personnages) et spatiale (multiplication et explorations des lieux) de la série.

S'intéresser au chronotope sériel nous oblige, plus que pour le cinéma, à souligner la nécessité d'une approche pragmatique, fondée sur la mise en évidence de l'expérience de la série comme processus. Dans cette perspective, les théories du world building proposent des pistes pour appréhender ces processus sériels comme appartenant à l'un des phénomènes majeurs de la culture médiatique contemporaine (Wolf, 2013). La définition de monde fictionnel la plus communément admise est celle de L. Doležel. Selon lui, un monde fictionnel possède trois propriétés : il est imaginaire - tout récit tisse, au fur et à mesure de sa progression, l'image d'un monde qui l'abrite; cohérent (dans la tradition aristotélicienne) - une propriété de ce monde et son contraire sont impossibles - et accessible - on peut le comprendre en se référant à la connaissance de notre propre monde grâce à certaines propriétés communes partagées par les deux : rapports humains (amour, pouvoir, désir, peurs), lois physiques, etc. ${ }^{2}$ (Doležel, 1985). Le désaccord théorique se cristallise cependant sur la consistance de ces mondes. Dans cette discussion, M.L. Ryan défend contre d'autres théoriciens des mondes possibles pour qui le monde ne se construit et n'existe que par le texte (U. Eco, L. Doležel, K. Walton), l'idée que le monde fictionnel est complet, c'est-à-dire qu'il dépasse ce qui en est décrit dans le texte et possède la même consistance que le nôtre ${ }^{3}$. Selon ce point de vue, l'exploration sérielle reviendrait à dissiper le brouillard obstruant les parties inconnues d'un monde fictionnel préexistant et complet à l'image du fog of war en jeu vidéo ${ }^{4}$. C'est cet espace à la croisée du concret et de l'abstrait qu'A.Gardies, cité plus haut, appelle dans les études cinématographiques «espace diégétique » et M.-L. Ryan (2012) dans les théories littéraires «univers narratif » que viennent se tisser les histoires et s'imbriquer les mondes. Comme un personnage à part entière, l'espace du monde fictionnel nous devient familier plongée après plongée, épisode après épisode. De cette double nature abstraite et concrète de l'espace filmique et de la spécificité sérielle qui permet au spectateur de replonger régulièrement dans le monde de la fiction, nait une sensation de familiarité.

M.-L. Ryan a contribué à l'approche spatiale des études fictionnelles en proposant des manières visuelles (via des cartes) de comprendre le chronotope, à la lumière des théories des mondes possibles. Cette inséparabilité entre le temps et l'espace elle la concentre dans son concept de storyworld:

Celui de storyworld est un concept facilement saisissable de façon intuitive, mais très difficile à définir d'une façon rigoureuse sur le plan théorique. La notion de monde suggère un espace, mais celle d'histoire évoque une séquence d'événements qui se développent dans le temps. Si nous concevons les storyworlds comme des représentations mentales construites pendant la lecture (ou visionnement, ou jeu, entre autres) d'un texte narratif, ils ne sont pas des conteneurs statiques pour les objets mentionnés dans les histoires, mais au contraire des modèles dynamiques de situations qui évoluent. Nous pourrions dire qu'ils sont des simulations du développement d'une histoire. Le critique russe Mikhail Bakthine (1981) a saisi l'inséparabilité de l'espace et du temps dans le concept de chronotope. (Ryan, 2013, p. 364 , notre traduction ${ }^{5}$.) 
Le storyworld est un espace dynamique et pluridirectionnel pouvant contenir des éléments temporels (récits et personnages) et appartenant à l'univers narratif qui peut en accueillir plusieurs. Pour les anthologies qui nous intéressent ici chaque épisode ou saison peuvent être analysés comme explorant de nouveaux storyworlds de l'univers narratif dans lesquels se déroulent différents récits et s'incarnent des personnages variés (Black Mirror, American Horror Story). Le cas de Fargo complexifie le modèle car certains éléments, objets ou personnages traversent les frontières de ces mondes narratifs, brouillant ainsi leurs limites. Toutefois, nous pourrions imaginer à des fins d'analyse, que le film constitue un storyworld et la série un autre. L'intérêt en est qu' « à la manière du chronotope de Baktine qui a pour fonction de concrétiser les rapports spatiotemporels en les rendant visibles, observables et par conséquent, analysables, le storyworld permet de rendre visible la métaphore des mondes de fiction et de les organiser par des schémas et des cartes. Le temps se matérialise dans l'espace (Bakhtine 1978, 391)» (Martinez, 2017, p. 56).

L'espace de la série se constitue ainsi au fur et à mesure des gestes que les consommateurs y développent: on aperçoit les génériques, qui ont la fonction de nous présenter un ensemble de promesses concernant cet espace; on choisit de s'immerger dans le monde, on le découvre au fil des épisodes par des processus de densification et d'immersion; on y revient à notre guise, pour des visionnages successifs. On va voir comment se construisent ces mondes, comment ils font naitre des histoires et comment ils deviennent de plus en plus étendus. Si, à ce stade de la réflexion, l'idée d'espace se révèle être pertinente pour l'analyse d'un objet télévisuel sériel, le défi reste encore de négocier avec sa « densité ».

\section{Dilater, meubler un espace au fil du temps sériel}

18 En adaptant le concept littéraire de monde à la série télé, J.-P. Esquenazi (2017, p. 161) identifie l'univers fictionnel d'une série comme base des récits sériels. Or, cet univers est souvent défini par une localisation spécifique qu'il nomme " centre de gravité » : l'hôpital universitaire de Cook County dans ER (NBC, 1994-2009) ; l'ile mystérieuse de Lost, (ABC, 2004-2010) et nous pourrions ajouter la maison familiale de Tony Soprano (HBO, 1999-2007), les bars respectifs de How I Met Your Mother (CBS, 2005-2014) et Friends (NBC, 1994-2004), le commissariat dans Hill Street Blues (NBC, 1981-1987), le motel dans Bates Motel (A\&E, 2013-2017), le lycée dans Glee (Fox, 2009-2015), etc. Ces espaces reconnaissables et spécifiques peuvent " être le décor unique ou presque de la série ou bien son centre de gravité, à laquelle s'ajoute un ensemble de personnages attachés à ce lieu ou qui ne font qu'y apparaître» (Esquenazi, 2017, p. 161). Par exemple, nous analysons à partir de cette définition, que toutes les actions des personnages de Game of Thrones (HBO, 2011-2019) - objet sériel spatial par excellence de par son générique cartographié évolutif et les enjeux territoriaux de ses intrigues - convergent vers King's Landing, centre de gravité de la série et lieu où est concentré le pouvoir. C'est la protection ou la conquête de ce lieu qui va lancer la narration et l'alimenter ${ }^{6}$. Remarquons à partir du concept de centre de gravité de J.-P. Esquenazi, que deux types d'espaces sériels se construisent autour de ces points: des espaces centrifuges et des espaces centripètes.

19 Les premiers sont des espaces dans lesquels on effectue des allers-retours depuis le centre de gravité. Ces allers-retours permettent de découvrir un territoire en multipliant les 
lieux visités au fil des épisodes. C'est le cas de Fargo dont les récits sillonnent saison après saison les alentours de la ville éponyme du Dakota du Nord, ou de Game of Thrones dans laquelle nous découvrons de nouvelles villes saisons après saison comme le rappelle le générique cartographié qui présente les lieux qui vont y être explorés dans l'épisode.

Les espaces centripètes, quant à eux, sont des espaces dans lesquels les situations liées à un centre de gravité et sa périphérie proche sont multipliées. Cette mécanique est utilisée dans les sitcoms: How I Met Your Mother (CBS, 2005-2014), Friends (NBC, 1994-2004); les séries hospitalières: ER, Dr House (Fox, 2004-2012), ou les teen series comme Glee qui construisent leurs récits en mille-feuille autour d'un (ou deux) lieux centraux. Dans les sitcoms ou comédies de situation par exemple, le traitement esthétique et narratif des espaces est lié au mode de production et de tournage, dans lequel, comme au théâtre, le public fait face à un décor. Entre les gradins et la scène, trois caméras filment trois axes différents faisant varier profondeurs de champ et échelles, du plan d'ensemble au gros plan. Les mouvements de caméra sont réduits aux panoramiques sur trépieds et aux travelings sur rails et le zoom à sa qualité numérique (le changement de lentille n'est possible qu'entre les scènes). La grammaire de ce type de productions tient généralement en une caméra qui isole un personnage, une autre qui capte la réaction d'un second (ou d'un groupe) et d'une troisième qui offre un angle plus grand pour mettre en contexte la situation narrative. La construction d'un espace unique, avec un nombre restreint de directions ${ }^{7}$, les plans nécessairement plus rapprochés, le nombre limité d'angles et la répétition dans les cadrages, sont autant de conséquences esthétiques liées au mode de production de ces programmes. Parmi ces espaces, certains sont si présents qu'ils deviennent l'égal des personnages qui le peuplent dans l'imagination du spectateur. C'est le cas du Central Perk, bar mythique de l'imaginaire de Friends et de son divan rose qui y flotte comme un radeau en son centre.

21 À l'inverse, ce que nous appelons les espaces centrifuges sont généralement produits selon un mode dit cinématographique : ils sortent des studios, utilisent des caméras plus mobiles et multiplient les lieux de tournages. Les conséquences de ce mode de production sur l'espace filmique sont notamment la multiplication des angles et la variation des échelles de plan avec l'apparition de l'establishing shot et du plan large. La conséquence narrative qui en résulte est une expansion de l'espace diégétique, phénomène qui comme nous l'avons vu est catalyseur de récits.

Le propre des séries serait donc de construire et dilater un espace en multipliant les lieux et les histoires qui les habitent et de le " meubler ${ }^{8}$ " sur le long terme si la série rencontre le succès auprès du public. Twin Peaks (ABC, 1990-91 puis Showtime, 2017), par exemple, se déroule autour d'une enquête policière, mais le parcours n'est pas linéaire. Il s'agit plutôt de la circulation du personnage de l'enquêteur, Dale Cooper, à travers les mystères que le petit village cache et à travers les multiples facettes de la réalité qu'il découvre et que les spectateurs découvrent avec lui. C'est dans ce sens que la révélation du coupable apparait bien avant la fin de la deuxième saison, rompant avec les règles télévisuelles d'un récit qui s'adapte à la grille et invitant les téléspectateurs à suivre une panoplie de personnages jusqu'alors considérés secondaires qui au contraire ne font que contribuer à épaissir les mystères. Nous suggérant qu'il y a toujours plus que ce que chaque épisode peut nous montrer, la série devient exploratoire. Cette exploration se produit au fur et à mesure de la progression de la série. 


\section{Pénétrer un espace fictionnel et s'y immerger périodiquement : le générique}

Dans le chapitre « Pouvoir des séries », J.-P. Esquenazi (2017, p. 78) souligne l'important pouvoir d'immersion des séries : «la série télévisée a, par sa durée, d'excellents atouts pour favoriser l'immersion téléspectatorielle. Une série télévisée est un texte fictionnel morcelé en de nombreux épisodes dont la diffusion s'étend sur une longue période de temps ». Les spectateurs renouent périodiquement avec le contrat fictionnel qu'ils ont passé avec la série. Si l'élément temporel (la répétition) joue un rôle important, l'effet d'immersion passe également par la construction d'un espace. Pour commencer, remarquons l'importance des génériques, territoires de négociation du contrat de la série, et élément qui peut permettre de comprendre comment celle-ci nous propose un espace à explorer selon un mode de narration potentiellement infini. Que ceux-ci soient longs (Games of Thrones, True Detective), courts (Girls, HBO, 2012-2017) ou constitués des premières images de l'épisode (Fargo), les génériques sont toujours le sas qui nous permet de pénétrer dans un monde, dans l'espace de la fiction. À la télévision le générique a deux fonctions précises, d'abord, il annonce le début du programme à un spectateur susceptible de ne pas être attentif. Ensuite, il permet d'introduire - et de réintroduire à chaque épisode - le spectateur dans l'univers et de lui faire découvrir - ou renouer avec le ton de la série. Généralement, il établit le contexte, l'environnement, les personnages et fonctionne comme une porte d'entrée vers la fiction. Le générique est aussi un des éléments par lesquels la série produit un milieu: ce qui se propose comme une exploration est un véritable processus de construction de mondes (voir l'analyse de Boni \& Re, 2017 ; Boni, 2017). La spécificité sérielle d'être propice à la densification d'un univers permet une immersion privilégiée.

Par exemple, remarquons que le générique de Twin Peaks ne présente pas ses personnages comme les séries chorales et téléromans de son époque. Au contraire, il fait vivre au spectateur une expérience immersive, par une longue divagation au sein d'un espace vidé de toute présence humaine. La séquence introductive s'ouvre sur l'image d'un oiseau qui disparait lentement en fondu et laisse apparaitre les rouages mécaniques d'une petite scierie rurale. Dans ces plans, les deux espaces (naturel et industriel) ont en commun de fonctionner seuls, comme régis par d'autres forces, que celles-ci soient-elles naturelles, surnaturelles ou mécaniques. S'ensuit un long plan fixe sur la route qui mène à la ville fictionnelle de Twin Peaks dans lequel la brume et les montagnes apparaissent calmes mais menaçantes. Cette sensation appuyée par la fixité du plan et son cadrage est consolidée par le survol d'une chute d'eau bouillonnante révélant tout à la foi sa beauté, sa force et l'unidirectionnalité immuable de son cours. La longueur des plans, les transitions lentes et magiques et l'imagerie somnolente donnent à l'univers une ambiance tranquille mais sinistre et anormale. La nature y a quelque chose de rassurant et d'hostile à la fois et influencera ses habitants ainsi que leur nature profonde.

L'espace magique de Twin Peaks se présente donc comme le substrat du récit par lequel vont être déterminées les actions, les paroles et l'apparence des protagonistes. Il est présent et attend d'être peuplé d'éléments temporels (personnages et récits). Ces deux minutes et demie introduisent le spectateur à la série et établissent son contexte et son environnement. C'est ainsi que le générique devient véritablement promesse et élément 
de séduction (Re, 2016), proposant un monde avec ses attraits, ses caractères thématiques et génériques, au bénéfice de spectateurs prêts (ou non) à embarquer dans l'expérience.

\section{Le pilote et la définition de l'espace}

Dans certains cas, le générique est inexistant et le titre et les crédits se superposent aux premières images de l'épisode. Or, pour J.-P. Esquenazi, plus que le générique, c'est le premier épisode tout entier qui donne le ton d'une série, esquisse ses soubassements, délimite la narration, l'univers fictionnel et le style de la série. C'est le cas de Fargo. Le premier épisode de la saison 1 s'ouvre sur un plan fixe et plongeant écrasant les objets, une voiture qui peine à émerger de la ligne d'horizon. L'homme n'est pas grand-chose dans cette nature hostile et l'horizon représente pour lui une barrière esthétique que l'on devine infranchissable. Dans les plans suivants, le noir et le blanc des premières images se teintent de rouge, le bas-côté de la route baigne dans la lumière des phares de la voiture. Cette contamination écarlate de la pureté blanche et du calme de la nuit noire annonce les évènements sanglants et inévitables qui vont suivre. Puis, des biches sauvages traversent la chaussée, provoquent la sortie de route du véhicule qui vient s'abimer au milieu d'un champ dans une explosion de neige.

Ces plans dépeignent un territoire figé, hostile, inhospitalier et dangereux qui se révélera être un opposant à part entière pour ceux qui peupleront la série. Cette scène introductive se termine sur un champ-contre-champ entre le conducteur rescapé et le cerf qui l'a percuté. Ce face-à-face annonce une lutte entre l'Homme et la nature, d'une part et, entre l'homme et sa propre nature de prédateur, d'autre part. Une analyse de la relation entre les personnages et leur environnement (social, géographique et familial) dans les deux premières saisons révèle que les personnages immoraux subissent leur espace tandis que les vertueux l'utilisent pour faire avancer leur enquête et rétablir la justice (Martinez, 2017, p. 64-67). Dans cette scène introductive sans dialogue, tous les éléments génétiques de la série s'inscrivent dans le paysage et peuvent donner lieu à une analyse esthétique ayant des répercussions sur l'analyse narrative.

\section{Fargo (FX, 2014-), une série spatiale?}

N. Hawley, le showrunner et principal scénariste de Fargo, décrit la série comme un long film de dix heures. Pourtant, on ne peut que constater que cet objet télévisuel non conventionnel selon son créateur', bénéficie bien d'une diffusion sérielle hebdomadaire classique sur la chaine Fox. À propos de son travail sur Fargo et Legion (FX, 2017-) il explique : «Nous avons le temps, non? Ce n'est pas un film de deux heures. C'est un film de 8, 10 ou 12 heures. Racontons donc les parties de l'histoire que nous ne pourrions pas raconter sur grand écran» (notre traduction ${ }^{10}$ ). Toutefois, par "partie» N. Hawley n'entend pas uniquement les récits mais certainement les lieux et recoins du Midwest coenien qu'il explore. Au huitième épisode de la deuxième saison, un gros livre relié de cuir s'ouvre et nous fait littéralement plonger (par un mouvement de caméra) dans un chapitre qui situe son récit à Luverne et est illustré d'une image d'Ed et Peggy, les personnages centraux de la saison (S1, E8 «The Castle»). Nous comprenons alors que chaque saison raconte un chapitre de ce livre intitulé "The History of True Crime in the Midwest ». Or, il s'avère que ces segments sont narrés les uns après les autres sans cohérence chronologique puisque la saison 1 se déroule en 2006, la 2 en 1978 et la 3 en 
2010 mais suivant une cohérence spatiale puisque chaque chapitre est annoncé par une identification de lieu du Midwest américain tel que «Luverne, Minnesota ». Mis bout à bout, les fragments d'univers narratif - le film étant considéré par N. Hawley comme l'un de ces fragment-chapitres - nous font découvrir le Midwest américain partiellement montré dans le film et son « histoire vraie » fictionnelle. Il s'agit d'une exploration qui est davantage spatiale que temporelle.

Cette omniprésence de l'espace par la mise en évidence de lieux nommés (Fargo, Brainerd, Bemdiji, Luverne, Sioux Falls) comme "fragment[s] sensible[s] d'espace[s] » (Gardies, 1993, p. 86) et de lieux symboliques comme le poste de police, le restaurant de route, le chalet dans la forêt, etc., engendre une dynamique narrative particulière. Dans tous les chapitres (film y compris) c'est ce Midwest coenien qui se pose généralement en principal opposant ou adjuvant aux personnages selon qu'ils sont femme ou homme défenseur de la loi ou bandit, citadin ou provincial.

L'analyse de la spatialité des séries par l'idéation d'un storyworld que l'on pénètre et que l'on visite au gré des épisodes met l'accent sur leur capacité à accueillir personnages et histoires pluridirectionnelles. La série Fargo exemplifie le fait que les histoires racontées naissent de paramètres spatiaux : ceux liés au Midwest américain. Avant la diffusion de la troisième saison, la chaine a choisi pour ses paratextes promotionnels de présenter l'espace et non les personnages (pourtant portés par des acteurs vedettes) ou les récits (à l'heure où il faut pourtant vite convaincre le spectateur au risque qu'il passe son chemin). De courtes vidéos teaser font passer l'espace en avant-plan des personnages. Y est figuré le milieu géographique et socioculturel nord-américain : on y voit la cour arrière d'une maison pavillonnaire ornée d'un père noël gonflable qui la dépasse en hauteur, l'intérieur d'un commissariat, une route enneigée sous la tempête, la devanture d'une pharmacie sous les flocons, etc. On y retrouve aussi l'image de l'oiseau comme dans le générique de Twin Peaks, perché cette fois-ci sur une banche enneigée. L'inhospitalité de l'environnement géographique sur fond de rêve américain donne à la série une saveur de «Sibérie avec des restaurants familiaux » dont parlaient les frères Coen à la sortie de leur film en 1996. On y met en avant la relation des individus à leur environnement, à leur quartier, à leurs valeurs, à leurs voisins et aux lois et le caractère anonyme d'une population enfouie dans d'épaisses parkas ou au fond de leurs voitures. La tyrannie de l'environnement géographique et socioculturel sera à l'origine de l'escalade vers l'irréversible atrocité. On note aussi qu'au sein même de l'espace fictionnel il y a les marques du genre (thriller, comédie, film policier, film noir) et du ton tragicomique et ironique des Coen. Ces fragments promotionnels constituent un paysage plus qu'un décor car, comme le note P. Leclerc (2011, p. 58) dans son mémoire sur l'espace dans les films de Terrence Malick, « les paysages "physiques" et "matériels” de tout film sont paysages en ce sens qu'ils participent à l'élaboration et à la construction mentale d'un paysage global, plus complexe, plus abstrait, d'une sorte d'“atmosphère" générale si on y tient ». D'où la notion de sensation d'espace qui est particulièrement intéressante si on observe la dimension transmédiatique de Fargo : les éléments spatiaux et narratifs du film de 1996 réapparaissant, déplacés, dans le récit récent de FX.

31 Le concept de monde se révèle être un bon outil pour étudier les espaces fictionnels car il permet d'analyser partiellement le phénomène d'expansion d'une série. Le monde est constitué non seulement de ce qui est montré mais également de ce qui ne l'est pas et de ce qui sera dévoilé. 


\section{Mondes tributaires de l'imagination du spectateur}

Les processus de construction et de densification d'un monde ne dépendent pas uniquement de ce qui est montré de ces mondes. Le monde demeure imaginaire et le spectateur participe largement à reconstituer de la continuité dans un espace découpé et non exhaustif. Or, même au cinéma ou en série télé, l'espace n'est pas nécessairement ce qui est donné à voir (c'est ce qui le différencie du lieu et du décor (Gardies, 1993). La série française Calls (Canal+, 2017) par exemple, se pose à l'ère de l'image, comme une expérience auditive au fort potentiel spatial puisqu'elle nous plonge dans un monde apocalyptique. Sa particularité est qu'elle ne montre de ce monde aucun paysage, misant sur le concept de found footage sonore. Les personnages y sont représentés par des voyants lumineux spatialisés dans l'image dont la présence et l'intensité lumineuse varient selon la localisation des protagonistes et l'intensité de leur voix. Leurs noms et conversations sous-titrent ces bouées visuelles et occupent l'image tout en cartographiant les interactions et déplacement des personnages. Cette série sonore - différente du podcast puisqu'elle est visuelle autant que sonore - mise sur l'imagination du spectateur et sa connaissance du monde réel - la convention cartographique qui place New York à gauche de l'océan atlantique et Paris à droite par exemple - pour construire le monde qu'elle projette. Celui-ci n'est plus seulement vu et entendu, il est visualisé et rend d'avantage compte de la sensation d'espace que des exemples figuratifs comme nous en avons l'habitude. Cette série sonore permet de manière paradoxale de mettre à jour le processus de création et d'élargissement d'un monde fictionnel sériel et de la mécanique narrative de la spatialisation en évacuant l'espace vu au profit d'un espace perçu.

\section{Mondes sériels}

L'espace construit par la série télé est un espace dans lequel nous entrons, que nous participons à construire, que nous visitons puis que nous quittons temporairement jusqu'à la prochaine visite. À l'image du parc de Westworld (HBO, 2016-), le monde fictionnel créé un monde imaginé et devient simulateur d'expériences. S'y immerger provoque le dépaysement et parfois, malgré les variations qu'impose la série, les spectateurs ressentent le besoin de retrouver cet espace, même si le décor est différent. Revenir dans un monde aimé est toujours possible : c'est ce que les séries semblent nous dire au travers des épisodes, des saisons, et ce même après un hiatus de plusieurs années comme pour Twin Peaks ou Roseanne (ABC, 1988-1997 puis ABC, 2018).

C'est également dans un sens spatial exploratoire que nous pouvons analyser les phénomènes récents de reboot, remake de séries ou de films qui deviennent des séries. Pensons par exemple Twin Peaks. The Return (Showtime, 2017) : la série nous propose une expérience solidement signée D. Lynch mais elle ne va pas retravailler le noyau narratif de l'enquête de Dale Cooper (en tout cas, si elle le fait ce n'est surement pas ce qui occupe la majorité du temps de la série). L'espace est ce qui émerge en toute sa force : il s'agit bien du monde de Twin Peaks, avec les lieux, mais aussi certains des personnages qui l'ont habité il y a 27 ans, désormais vieillis. Revenir à Twin Peaks est une façon d'habiter à nouveau, d'explorer à nouveaux frais, ce monde complexe. C'est le cas également de Fargo qui prolonge l'univers du film sans en proposer un remake (les personnages et les récits 
sont différents), un reboot (les temporalités se superposent et ne se remplacent pas). La notion d'exploration spatiale est une bonne alternative à celle de réécriture.

\section{Conclusion}

Nous avons mis l'accent sur la dimension pragmatique de l'expérience du récit, en nous intéressant au phénomène de la sérialité télévisuelle. Par l'appui sur les limites d'une logique temporelle, fondée sur la grille des programmes classique avec ses intervalles et ses saisons qui, à l'ère du streaming, n'est plus la dominante, ainsi que par une lecture s'appuyant sur les théories de mondes fictionnels, nous avons remarqué la pertinence d'une approche spatiale, valide surtout lorsqu'on tient compte des anthologies et des nombreux reboots et remakes qui nourrissent les vastes franchises médiatiques dont les séries font partie. Comme on l'a vu dans les quelques exemples discutés, l'espace est souvent vecteur de la narration et, de manière plus large, l'espace quitte son statut de simple décor pour devenir un monde explorable dont la découverte dépend tout autant de la série elle-même que de ses spectateurs, de leurs pratiques et de leur travail cognitif. Penser les séries comme des expériences de construction de mondes est donc une piste permettant de comprendre aussi les mécanismes de succès d'une œuvre sérielle et d'analyser les enjeux de sa longévité, concept qui représente encore un défi pour l'analyse.

\section{BIBLIOGRAPHIE}

BAKHTINE, M. (1978) [1975]. Esthétique et théorie du roman. Trad. du russe par D. Olivier. Paris : Gallimard.

BONI, M. (2017). « Psycho/Bates Motel : hyperdiégèse et réactivation sélective ». Intermédialités 28-29. En ligne : https://www.erudit.org/fr/revues/im/2016-n28-29-im03201/1041077ar/.

BONI, M. \& BERTON, M. (à paraitre). « Comment étudier la complexité des séries télévisées ? : problèmes et méthodes ", TV/Series.

BONI, M. \& RE, V. (2017). « Here Be Dragons. La mappa come soglia, racconto, creazione ». In : BONI, M. \& RE, V. (éds), Game of Thrones. Una mappa per immaginare mondi. Milano/Udine : Mimesis, p. $105-128$.

DOLEŽEL, L. (1985). « Pour une typologie des mondes fictionnels ». In : Parret, H. \& Ruprecht, H.-G. (dirs), Exigences et perspectives de la sémiotique. Recueil d'hommages pour A. J. Greimas. Amsterdam : John Benjamins, p. 7-23.

DUBoIS, F.-R. (2015). « Organisation proleptique et clôture narrative dans les séries télévisées ». Écrans 4, p. 110-120.

Eco, U. (1985) [1979]. Lector in fabula. Le rôle du lecteur, ou, La Coopération interprétative dans les textes narratifs. Trad. de l'italien par M. Bouzaher. Paris : Librairie générale française. 
ELLIS, J. (2011). « Interstitials: How the "Bits in Between" Define the Programmes ». In: Grainge P (ed.), Ephemeral Media: Transitory Screen Culture from Television to YouTube. Basingstoke : Palgrave Macmillan, p. 59-69.

ESQUENAZI, J.-P. (2014) [2010]. Les Séries télévisées. L'avenir du cinéma? Paris : Armand Colin.

ESQUENAZI, J.-P. (2017). Éléments pour l'analyse des séries. Paris : L'Harmattan.

GARDIES, A. (1993). L'Espace au cinéma. Paris : Méridiens Klincksieck.

GAUdREAUlt, A. \& JOST, F. (2017). Le Récit cinématographique. Films et séries télévisées. Paris : Nathan.

JOST, F. (2015). Les Nouveaux Méchants. Quand les séries américaines font bouger les lignes du bien et du mal. Montrouge : Bayard.

LECLERC, P. (2011). Le Traitement de l'espace dans Badlands et Days of Heaven, de Terrence Malick. Mémoire de maitrise : Université de Montréal.

LIFSCHUTZ, V. (2018). This is the End. Tours : Presses universitaires François-Rabelais.

MARTIN, B. (2014) [2013]. Des hommes tourmentés. Le nouvel âge d'or des séries. Trad. de l'anglais par L. Cohen. Paris : Éditions La Martinière.

MARTINEZ, C. (2017). De Fargo à Fargo (Coen 1996 ; FX 2014-) : la notion d'espace dans un phénomène médiatique complexe, l'adaptation d'un film en série télévisée. Mémoire de maitrise : Université de Montréal.

mittell, J. (2006). « Narrative Complexity in Contemporary American Television ». Velvet Light Trap 58. p. 29-40.

MitTelL, J. (2015). Complex TV: The Poetics of Contemporary Television Storytelling. New York : New York University Press.

PESCATORE, G. \& INNOCENTI, V. (2012). «Information Architecture in Contemporary Television Series ». Trad. de l'italien par S. et M. Francoli. Journal of Information Architecture 4 (1-2), p. 57-72.

RE, V. (2016) « From Saul Bass to Participatory Culture: Opening Title Sequences in Contemporary Television Series ». Necsus. En ligne : https://necsus-ejms.org/saul-bass-participatory-cultureopening-title-sequences-contemporary-tv-series/.

RYAN, M.-L. (2012). « Space ». In : Hühn, P. et al. (dirs), Handbook of Narratology. Berlin/New York : De Gruyter, En ligne : http://www.lhn.uni-hamburg.de/article/space.

RYAN, M.-L. (2013). « Transmedial Storytelling and Transfictionality ». Poetics Today 34 (3), p. 361-388.

SAINT-GELAIS, R. (2011). Fictions transfuges. La transfictionnalité et ses enjeux. Paris : Éditions Le Seuil.

SOULEZ, G. (2011). « La double répétition : structure et matrice des séries télévisées ». Mise au point 3. En ligne : https://journals.openedition.org/map/979.

WOLF, M. J.P. (2013). Building Imaginary Worlds: The Theory and History of Subcreation. New York : Routledge. 


\section{NOTES}

1. Concernant la mémoire collective et les communautés de savoirs («intelligence collective ») au service de la complexification voir aussi Mittell (2006, p. 31).

2. Certaines lois de notre monde peuvent être transgressées par les mondes fictionnels. Dans ce cas, les spécificités de ces mondes doivent être exposées comme règles admises dès leur exposition sous peine de devenir incohérentes par la suite. C'est ainsi que nous pouvons accepter la présence de magie dans des mondes fictionnels (M.-L. Ryan).

3. La notion de complétude et d'incomplétude des mondes textuels divise L. Doležel, U. Eco et K. Walton, d'une part, et T. Pavel, M.-L. Ryan et J. Woods d'autre part. Un bref historique de la discussion est présenté dans Fictions transfuges (Saint-Gelais, 2011, p. 49-70).

4. Le fog of war est la formule utilisée par les joueurs pour décrire les zones occultées sur une carte dans des jeux tels que Civilization, Warcraft, Starcraft, etc. L'espace s'y découvre à mesure que les personnages les traversent.

5. «Storyworld is a concept that makes a lot of intuitive sense, but it is very difficult to define in a theoretically rigorous way. World suggests a space, but story is a sequence of events that develops in time. If we conceive of storyworlds as mental representations built during the reading (viewing, playing, among others) of a narrative text, they are not static containers for the objects mentioned in a story but rather dynamic models of evolving situations. We could say that they are simulations of the development of the story. The Russian critic Mikhail Bakhtin (1981) captured this inseparability of space and time in narrative through the concept of chronotope. "

6. Il s'agit de l'un des trois lieus avec Castle Black (lieu principal de la résistance) et le Mur (lieu d'où provient la menace à long terme) à persister au générique, dont la particularité est d'évoluer en fonction des territoires explorés.

7. Dans le studio de tournage de Friends le décor dissimule une ouverture dans l'un des murs de l'appartement afin d'offrir un angle supplémentaire camouflable, masqué par un tableau appartenant au décor.

8. L'idée de meubler un monde est attribuable à U. Eco (1985, p. 158).

9. Dans une interview donnée à Vanity Fair en 2016 il dit «Let's not rely on all the old formulas. Let's not have a case of the week» (http://vanityfair.com).

10. "We've got the time, right? It's not a two-hour movie. It's an 8- or a 10- or a 12-hour movie. Let's tell the parts of the story that you couldn't tell on the big screen. "

\section{RÉSUMÉS}

Partant d'une approche pragmatique de la réception qui considère la série télévisée comme processus et expérience exploratoire d'un espace diégétique pluridirectionnel, cet article allie analyse esthétique et narratologique en comparant les séries télévisées à des mondes fictionnels. 
À travers divers exemples de programmes des années 1990 à aujourd'hui, l'objectif est de penser les séries comme des expériences de construction de mondes et de comprendre les mécanismes de leur pouvoir immersif sur le long terme. L'espace, conçu en tant que monde ou territoire diégétique, permettra de compléter les méthodes d'analyse des séries télévisées présentant le temps comme moteur narratif principal et s'appuiera sur les théories spatiales des études cinématographiques et médiatiques.

This contribution puts forward a spatial perspective to the study and analysis of television series. Starting from a pragmatic approach, that considers the television series as a process and an exploratory experience of a multidirectional diegetic space, the point of view that we adopt here combines aesthetic and narratological analysis and compares television series to fictional worlds. Through various examples of shows from the 1990s to today, this essay displays series as worldbuilding experiences and tries to understand the mechanism of their immersive power in the long run. Working on spatial theories of film and media studies we conceive space as a world or a diegetic territory, in order to complete the existing methods for the analysis of television series that consider time as the main element of a serial narrative.

\section{INDEX}

Keywords: TV series, space, fictional worlds, narrative exploration, immersion, narrative expansion, longevity, titles, pilot

Mots-clés : série télévisée, espace, mondes fictionnels, exploration narrative, immersion, expansion narrative, longévité, générique, épisode pilote

\section{AUTEURS}

\section{MARTA BONI}

Université de Montréal, CA-H3C 3J7 Montréal (Québec), Canada

\section{CAMILLE MARTINEZ}

Université de Montréal, CA-H3C 3J7 Montréal (Québec), Canada 